\title{
Research on Smart Agriculture Cloud Platform Based on Internet of Things Technology
}

\author{
Ke Yao, and Qiang Wang \\ Shandong Vocational College of Industry, Zibo 256414, China
}

Keywords: Internet of things, Smart agriculture, Cloud platform

\begin{abstract}
The Internet of Things technology plays an important role in changing the production mode of traditional agriculture. It can promote the intelligent development of agriculture and use resources more efficiently. In the current agricultural production, cloud platforms play a positive role in building smart agriculture, and the Internet of Things is used to systemize agricultural production. Based on this, this article starts with an overview of smart agriculture, first analyzes the application of the Internet of Things technology in smart agriculture, and then explores the construction of a smart agricultural cloud platform based on the Internet of Things technology, hoping to provide a certain amount of research on the construction of modern agricultural cloud platforms Reference comments.
\end{abstract}

\section{Introduction}

China is rich in land resources and is also a large agricultural country. However, from the current actual situation, China's agricultural production still adopts a more traditional model, which not only causes waste of fertilizer and water resources in fertilization and irrigation, but also reduces agricultural planting Yield. Therefore, in recent years, many regions in my country have carried out the upgrade of agricultural production, using the Internet of Things technology to build a smart agricultural cloud platform to provide a full range of services for agricultural production, so as to enhance the production capacity of land resources.

Smart agriculture is an important measure to reduce the gap between the rich and the poor and to promote the development of agricultural economy. It uses the Internet of Things technology to control the entire process of agricultural production, and it can also meet the functions of remote diagnosis and disaster warning, which can provide the ultimate for modern agricultural production. Great help. At present, most of my country's agricultural production relies on the experience of workers, and there is no scientific system. With the help of smart agriculture, it can provide modern technical guidance for agricultural production, promote the upgrading of agricultural production structure, and increase the income of farmers. From the perspective of composition, the current smart agriculture mainly includes computer technology, communication technology and sensor technology, which can realize the monitoring of the agricultural production process, while ensuring that agricultural production will not cause greater pressure on the ecological environment [1].

Since the reform and opening up, my country's agriculture has developed rapidly, and output has greatly increased. However, in the process of agricultural production, there is a problem of fertilizer and pesticide abuse, which has caused greater pressure on the environment. In addition, due to the adoption of traditional business models, the competitiveness of agricultural production is weak, so smart agricultural production has attracted more and more attention. Smart agriculture can also improve the operating efficiency of agricultural production, use intelligent machinery to replace traditional manual labor, ease the problem of labor tension, realize intensive agricultural production, improve agricultural e-commerce system, and completely change the traditional low-tech content situation. 


\section{Application of Internet of Things Technology in Smart Agriculture}

\subsection{Smart Agriculture Supervision}

In the smart agriculture system, the Internet of Things technology should realize the supervision of the agricultural production process, and strengthen the safety information management of agricultural products while collecting the growth status of crops, so that the growth of crops can be analyzed more accurately, and subsequent management activities can be carried out. [2]. Specifically, in the supervision of smart agriculture, the Internet of Things technology is mainly applied in the following aspects:

(1) Disease prevention. In agricultural production, various crop diseases have a greater impact, small ones can cause problems such as production reductions, and large ones can cause serious economic losses, greatly increasing the risks of agricultural production. In smart agriculture supervision, the Internet of Things technology will collect the growth status of crops to determine whether there are diseases and achieve effective protection.

(2) Fertilization management. The Internet of Things technology can analyze the various elements needed by crops according to their growth conditions and collect information on the content of various elements in the soil. This can provide effective guidance for subsequent fertilization, on the one hand, reducing the amount of fertilizer used, on the other hand Reduce the impact of fertilization on the ecological environment.

(3) Inspection of crop growth environment. The growth of crops requires a suitable environment, which is the key to ensuring yield. Through the Internet of Things technology, it is possible to monitor the production environment of crops, analyze the heavy metals, acidic substances, etc., and then intelligently regulate them to ensure the healthy growth of crops.

\subsection{Smart Agricultural Resource Management}

Modern agricultural production is inseparable from the use of resources. Especially for crops, watering and fertilizing can be targeted according to their characteristics, which can effectively increase crop yields. In traditional agricultural production, fertilization and watering mostly rely on farmers' experience, which will not only cause waste of resources, but also increase the pressure on the ecological environment [3]. In smart agriculture, the Internet of Things technology can use remote sensing and Internet technologies to analyze the condition of the soil and judge the growth of crops. It can not only provide guidance for fertilization and watering, but also realize pest control and modern agricultural production resource management.

In traditional agricultural production, it is very difficult to collect crop information, so it is impossible to judge the scientific parameters of fertilization and watering. However, the Internet of Things technology can solve this problem well, effectively integrating multiple technologies to realize digital agricultural production the use of resources has significantly improved the scientific nature of agricultural production.

\subsection{Refined Operation of Smart Agriculture}

Refined operation is an important part of smart agriculture, using modern technology to optimize production methods, liberate labor, and improve the efficiency of agricultural production [4]. In specific applications, the Internet of Things technology can upload various types of information about agricultural production to achieve data integration and efficient use, so that it can promote intelligent agricultural production and achieve intensification of production. In addition, in the process of agricultural production, the Internet of Things technology can also plan sophisticated agricultural production plans through big data analysis, and provide scientific guidance for agricultural production activities such as cultivation and irrigation.

Refined operation is an important manifestation of modern agricultural production. It is no longer the traditional extensive management, but the most appropriate method for specific crop conditions. This requires the use of IoT technology to effectively analyze the collected data and analyze the most appropriate refined operation method. 


\subsection{Smart Agricultural e-Commerce}

In traditional agriculture, sales mostly rely on specific channels, which are easily affected by various factors, and problems of unsalable sales often occur, especially for seasonal fruits and vegetables. Once sales are blocked, farmers' Economic income has a greater impact. In smart agriculture, the Internet of Things technology can provide rich marketing ideas for agricultural products, and achieve rapid sales through e-commerce channels [5]. In addition, due to the introduction of the Internet of Things technology, the production and circulation of agricultural products are more transparent, which can also provide a guarantee for the quality of the products. Judging from the current actual situation, the platform built by the Internet of Things technology can also supervise the entire process of agricultural production, thereby enhancing the trust of consumers and providing strong support for the sales of agricultural products.

Judging from the current actual situation, people pay more attention to the quality and safety of products when shopping online, especially for agricultural products. Consumers want to know the production process of the product to determine whether they meet their needs. Through the construction of smart agriculture, it can effectively meet the e-commerce needs of modern agricultural production and provide consumers with real information so that they can directly observe the process of agricultural product production, thereby stimulating consumption, gradually forming a brand, and increasing farmers' income.

\section{The Construction of a Smart Agricultural Cloud Platform Based on the Internet of Things Technology}

\subsection{System Architecture}

Smart agriculture must incorporate a variety of modern technologies to not only realize the collection of crop information, but also realize functions such as management optimization and disaster prevention. Therefore, the cloud platform for smart agriculture must include thermal imaging dome cameras, Ethernet communication networks, smart irrigation systems, user terminals, display screens, and weather stations, which are integrated into the smart agriculture cloud platform to realize smart agricultural production management, The specific only agricultural bank cloud platform system architecture is shown in Figure 1.

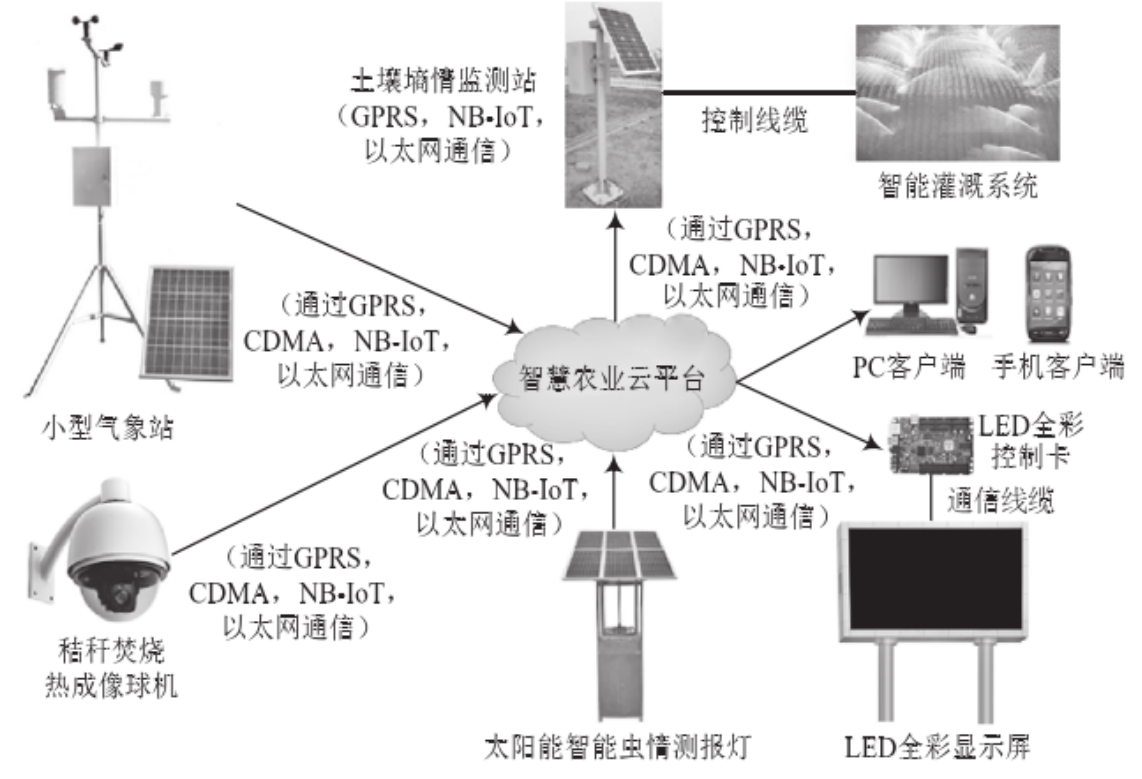

Figure 1 System Architecture Diagram of Smart Agriculture Cloud Platform

\subsection{Infrastructure}

In order to effectively realize the functions of the smart agricultural cloud platform, its infrastructure needs to be improved. Judging from the specific situation of current agricultural 
production, the smart agricultural cloud platform should include the following infrastructure:

(1) Meteorological environment information monitoring system. The meteorological environment information monitoring system is mainly realized with the help of small weather stations to monitor the meteorological environment in a small area, collect information such as humidity, wind direction, wind speed, and temperature, and then feed the information back to the data processing center of the cloud platform. As shown in Figure 1 above, the collected information can be fed back to the user terminal or display screen, so that the user can understand the collected information in time.

(2) Soil monitoring and remote irrigation control. Soil monitoring mainly collects soil moisture and temperature information, then uploads the data to the Internet of Things system, and determines whether to perform irrigation operations through a preset program. Generally speaking, the irrigation system of the smart agricultural cloud platform can be divided into automatic control and manual control, and can be switched at will, and the user can control it on the terminal. For example, when the soil moisture content is low, the user can remotely control the watering system switch on the computer, and turn off the irrigation system when the soil moisture meets the requirements.

(3) Visual monitoring system. The visual monitoring system is one of the important channels for all-round monitoring of the agricultural production process. It uses the Internet, cameras, and servers to collect information, and can store relevant information in the server. At this time, people can check relevant pictures or video data through the Internet to judge the growth of crops. Generally speaking, access to visual monitoring systems requires permissions, and users can set relevant login permissions according to their own needs.

\subsection{Iot Platform}

The Internet of Things is the basis for the construction of a smart agricultural cloud platform. It connects various devices to collect data in a specific environment, and at the same time organizes the data and transmits it to the processing system, and then performs various operations through feedback. Through the Internet technology, various parameters in agricultural production can be effectively collected, and then the terminal module can be used to perceive the corresponding demand, and the overall data can be tracked and recorded, and then transmitted through the network, which can effectively control the overall agricultural production. The process is controlled. In addition, the use of IoT technology to build a smart agricultural cloud platform can also effectively provide early warning. When certain parameters are found to be abnormal, users will be provided with corresponding early warning information, so that the impact of emergencies on agricultural production can be better controlled [6].

When building a smart agricultural cloud platform, the function required by the Internet of Things is to collect and transmit data, so it mainly depends on various sensor equipment in the agricultural base. When the device collects data, it can be transmitted to the agricultural cloud platform through the network, and then the entire agricultural production will be linked together as an intermediate bridge for transmission. In this way, many drawbacks in traditional agricultural planting can be effectively changed, the timely transmission of information can be realized, the information can be responded to in the shortest time, and the agricultural production can be managed by intelligent means to improve management efficiency.

\section{Conclusion}

The smart agricultural cloud platform plays an important role in modern agricultural production, which can effectively improve the efficiency of agricultural production and at the same time improve the utilization rate of various resources. In the construction of a smart agricultural cloud platform, the Internet of Things technology is indispensable. It can effectively combine various equipment and technologies to realize the collection and transmission of information, and facilitate agricultural producers to control the process of agricultural production as a whole. Based on this, this article first analyzes the application of Internet of Things technology in smart agriculture, divided into smart agricultural supervision, smart agricultural resource management, smart 
agricultural refined operation and smart agricultural e-commerce to explore four aspects, and then research based on the Internet of Things technology The construction of the intelligent agricultural cloud platform of China is summarized from the three aspects of system architecture, infrastructure and IoT platform. It is hoped that this can provide a certain reference for the improvement and perfection of China's intelligent agricultural cloud platform.

\section{Acknowledgment}

The paper is the phased research result of the Zibo City's key research and development plan “Zishui Online” smart water IoT cloud service platform (project number: 2019ZBXC246).

\section{References}

[1] Lu Linfeng, Guan Xiaofeng, Huang Hailong, et al. Application platform construction based on agricultural Internet of Things [J]. Zhejiang Agricultural Sciences, vol.61, no.7, pp.1455-1457, 2020.

[2] Shen Weiwei, Zhou Bo. Research on the development context and key areas of smart agriculture [J]. Green Science and Technology, no.08, pp.192-195+214, 2020.

[3] Sun Shu. Research on smart agriculture and its key technologies based on the Internet of Things architecture [J]. Science and Technology Innovation, no.14, pp.78-79, 2020.

[4] Yang Weimin, Pan Qingxian, Huang Sujuan. Smart agriculture opens a new chapter in agricultural modernization-the application of Internet of Things technology to develop smart agriculture in Puyang Street, Pujiang County [J]. Modern Agricultural Machinery, no.2, pp.4-5, 2020.

[5] Yin Juan. Design and development of smart agricultural precision planting system driven by the Internet of Things [J]. Journal of Jinling Institute of Technology, vol.36 no.1, pp.88-92, 2020.

[6] Gabriel McNunn, Douglas L. Karlen, William Salas, et al. Climate smart agriculture opportunities for mitigating soil greenhouse gas emissions across the US Corn-Belt[ J]. Journal of Cleaner Production, no.24, pp.41-50, 2020.

[7] Tian Fuzhong, Wang Yilei, Wang Dexin, et al. The application of Internet of Things and artificial intelligence technology in smart agriculture [J]. Rural Economy and Technology, vol.31 no.5, pp.14-16, 2020. 